\title{
Gallstone Ileus with Cholecysto-Gastric Fistula in an Older Female.
}

\author{
Roger Taylor ${ }^{1}$, Nnennaya Opara ${ }^{1}$, and Taylor Simmerman ${ }^{1}$ \\ ${ }^{1}$ Affiliation not available
}

February 15, 2022

Roger S Taylor DO ${ }^{1}$, Nnennaya U Opara MD, MPH ${ }^{1,2}$, Taylor Simmerman DO ${ }^{1}$

${ }^{1}$ Emergency Medicine, Charleston Area Medical Center, Charleston, USA

${ }^{2}$ Institute for Academic Medicine, Charleston, TX, USA

Corresponding authors : Nnennaya U Opara, Nnennaya.opara@camc.org Charleston Area Medical Centre 3200 MacCorkle drive.SE Charleston, WV 25304

\section{Introduction}

According to the Centers for Disease Control and Prevention (CDC), cases of non-injury emergency department (ED) visits for which abdominal pain was the chief complaint increased by $31.8 \%$ from 2000 (5.3 million) to 2008 (7 million), constituting a $7.6 \%$ rise, and continue to increase [1]. Small bowel obstructions account for $2 \%$ of all patients presenting to the ED with abdominal pain [2] and may have many different etiologies, although the most common is tissue adhesions from previous abdominal surgeries, followed by hernias [3]. Herein, we present a case of abdominal pain secondary to a rare cause of bowel obstruction.

\section{Case presentation}

A 70-year-old Caucasian woman presented to the ED with the chief complaint of abdominal pain. Symptoms had started two days prior with intermittent dull abdominal pain, constipation, and dysuria. Over-thecounter laxatives seemed to alleviate constipation at that time; however, the other symptoms worsened, prompting the patient to meet with her primary care physician (PCP). She denied nausea or vomiting. The patient's medical history was negative for surgery and she denied the use of tobacco, alcohol, or illicit drugs. Her family history was non-contributory. The PCP ordered a plain abdominal X-ray, which showed a possible small bowel obstruction, and the patient was immediately advised to visit the ED for further evaluation.

\section{Investigations}

Physical examination revealed mild pain on palpation of the entire abdomen. Computed tomography (CT) of the abdomen and pelvis with intravenous (IV) and oral (PO) contrasts revealed a 2.7-cm gallstone in the terminal ileum with dilated loops of the small bowel in the right lower quadrant, indicative of gallstone ileus [fig1]. 


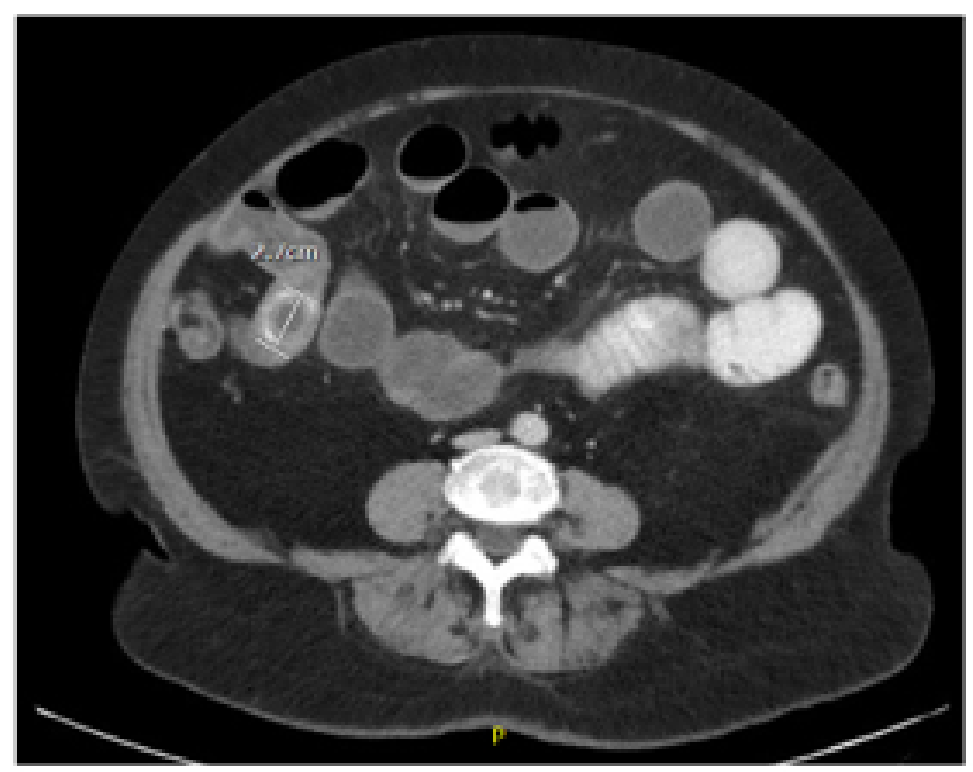

Pneumobilia was equally evident with PO contrast in the contracted gallbladder, suggesting a cholecystogastric fistula (fistula between the gallbladder and gastric antrum) [fig 2].

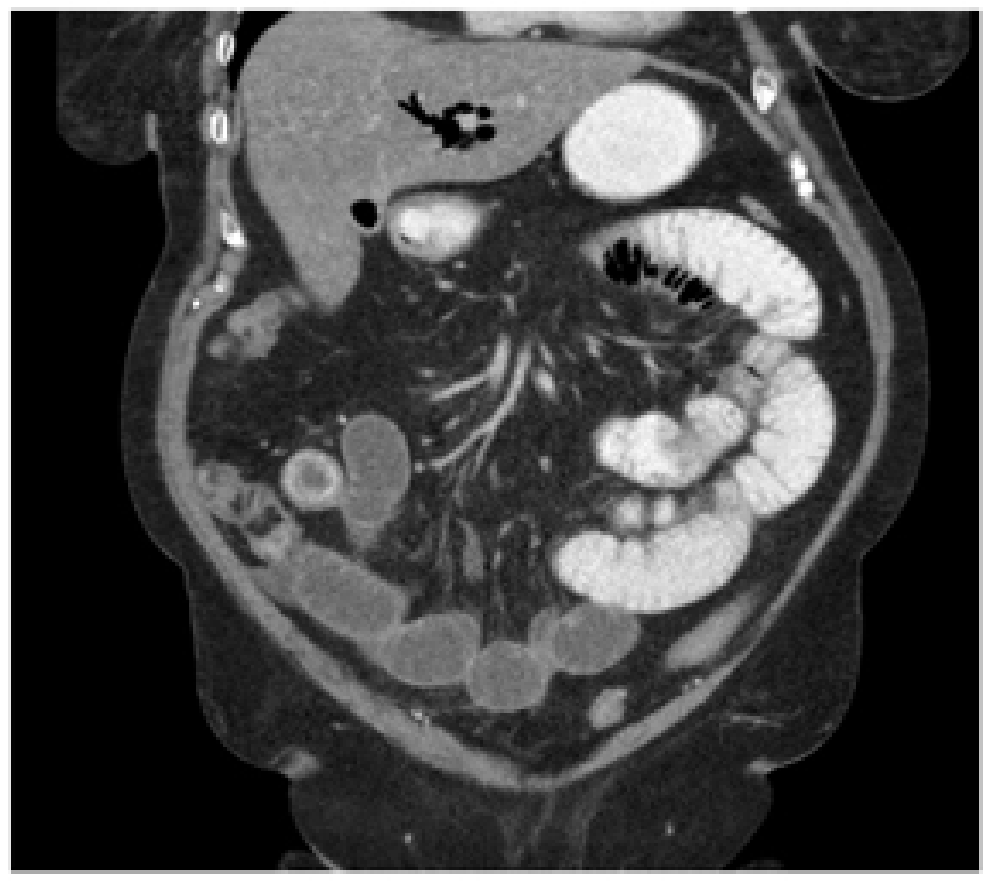

Peptic ulcer with perforation was subsequently ruled out based on the PO contrast scan result as well as the 
lack of clinical symptoms of peptic ulcer in our patient.

\section{Treatment}

A general surgery consultation was obtained. The patient was treated with a nasogastric tube and IV fluids and admitted to the general ward. After symptomatic treatment, the patient developed severe abdominal pain with worsening of symptoms and was immediately transferred to the operating room for exploratory laparotomy with manual extraction of the stone by an assisted enterotomy.

Outcome and Follow-up

On post-operative day one, the patient was able to pass the flatus and, on day two, she moved her bowels. The patient recovered well, and a decision was made to defer cholecystectomy and fistula closure.

\section{Discussion}

Adhesions continue to be the most common cause of small-bowel obstruction, as has been noted previously [3]. Gallstone ileus is a rare condition. The incidence is approximately $0.095 \%$ of all causes of mechanical bowel obstruction. Gallstone ileus primarily affects older females [4]. Unfortunately, patients with gallstone ileus often present only vague complaints. Patients commonly experience symptoms of episodic abdominal pain due to a dislodged gallstone, which intermittently/partially blocks the cystic duct until it is completely blocked [5]. Some patients have complained of nausea and vomiting, although others have experienced more severe symptoms. The most common distal site of obstruction is the ileocecal junction [6]. However, gallstones can obstruct any part of the intestinal tract, including the proximal gastric outlet, a phenomenon known as Bouveret syndrome [7].

When gallstone ileus is diagnosed, it is imperative to thoroughly examine the entire biliary tree to identify the exit point of the gallstone. Common pathways of gallstones exiting the gallbladder due to fistula formation include cholecysto-duodenal (75\%), cholecysto-colonic (10\%-20\%), and cholecysto-gastric (5\%) [8].

Chronic cholecystitis is the main cause of fistula formation [9]. The definitive treatment for fistula formation is surgery, during which manual extraction of the gallstone with an assisted enterotomy is performed, with either cholecystectomy with fistula closure, bowel resection, or bowel resection with fistula closure [10]. However, most fistula formation cases are treated by enterotomy and stone extraction alone. No randomized clinical control trials have evaluated these options to date, but this is likely due to the low incidence rate of gallstone ileus.

\section{References}

(1) F. Bhuiya, S.R. Pitts, and L.F. McCaig. "Emergency department visits for chest pain and abdominal pain: United States, 1999-2008," NCHS Data Brief , no 43. Hyattsville, MD: National Center for Health Statistics, 2020.

(2) M.R. Taylor and N. Lalani. "Adult small bowel obstruction," Academic Emergency Medicine , vol. 20, no. 6 , pp. 527-544, 2013. doi:10.1111/acem.12150

(3) D. Menzies and H. Ellis. "Intestinal obstruction from adhesions-how big is the problem?" Annals of the Royal College of Surgeons of England, vol. 72, no. 1, pp. 60-63, 1990.

(4) W.J. Halabi, C.Y. Kang, N. Ketana, et al., "Surgery for gallstone ileus: A nationwide comparison of trends and outcomes," Annals of Surgery, vol. 259, no. 2, pp. 329-335, 2014. doi:10.1097/SLA.0b013e31827eefed

(5) C.M. Townsend, R. D. Beauchamp, B.M. Evers, and K.L. Mattox. Sabiston Textbook of Surgery: The Biological Basis of Modern Surgical Practice. 18th edition. Philadelphia, PA: Elsevier Saunders, 2008.

(6) N. Williams, P.R. O'Connell, et al., Bailey \& Love's Short Practice of Surgery . 26th edition. CRC Press, 2013. 
(7) G.P. Ramos and N.E. Chiang NE. "Bouveret syndrome," New England Journal of Medicine, vol. 378, no. 14 , p. 1335 , 2018. doi: 10.1056/NEJMicm1711592

(8) C. Hernandez, D. Heuman, and Z.R. Vlahcevid. "Pathophysiology of disease associated with deficiency of bile acids," Principles and Practice of Gastroenterology and Hepatology, New York: Elsevier Science, pp. 384-395, 1998.

(9) S.L. Summerton, A.C. Hollander, J. Stassi J, et al., "US case of the day. Gallstone ileus," Radiographics , vol. 15, no. 2, pp. 493-495, 1995.

(10) M. Van-Hillo, J. Van- der- Vilet, T. Wiggers, et al., "Gallstone obstruction of the intestine: An analysis of ten patients and a review of the literature," Surgery, vol. 101, no. 3, pp. 273-276, 1987.

\section{Data Availability Statement:}

All data used in the study is cited within the manuscript

Conflict-of-Interest Statement:

Authors have no competitive interest to declare.

Funding Statement:

There was no funding provided for this study. 